\title{
Duloxetine in the treatment of chronic pain due to fibromyalgia and diabetic neuropathy
}

This article was published in the following Dove Press journal:

Journal of Pain Research

15 December 2010

Number of times this article has been viewed

\author{
Alan Wright \\ Kyle E Luedtke \\ Chad VanDenBerg \\ Center for Clinical Research, Mercer \\ University, Atlanta, Georgia, USA
}

\begin{abstract}
Duloxetine is a serotonin-norepinephrine reuptake inhibitor approved by the US Food and Drug Administration for the treatment of fibromyalgia and painful diabetic neuropathy at doses of $60 \mathrm{mg}$ daily. Duloxetine has been shown to significantly improve the symptoms of chronic pain associated with these disorders, as measured by the Fibromyalgia Impact Questionnaire, Brief Pain Inventory scores, the Clinical Global Impressions Scale, and other various outcome measures in several placebo-controlled, randomized, double-blind, multicenter studies. Symptom improvement generally began within the first few weeks, and continued for the duration of the study. In addition, the efficacy of duloxetine was found to be due to direct effects on pain symptoms rather than secondary to improvements in depression or anxiety. Adverse events including nausea, constipation, dry mouth, and insomnia, were mild and transient and occurred at relatively low rates. In conclusion, duloxetine, a selective inhibitor for the serotonin and norepinephrine transporters, is efficacious in the treatment of chronic pain associated with fibromyalgia or diabetic neuropathy, and has a predictable tolerability profile, with adverse events generally being mild to moderate.
\end{abstract}

Keywords: duloxetine, chronic pain, neuropathic pain, fibromyalgia, efficacy, safety

\section{Introduction}

Chronic pain is a serious and debilitating condition with a large financial burden to the suffering individual, their family, and society. The etiology of chronic pain can be due to a multitude of factors, from malignant disease to diabetic neuropathy. Whatever the cause, chronic pain is highly stressful for the patient, and can lead to additional psychiatric and somatic conditions (Table 1). ${ }^{1}$ Treatment of chronic pain using established interventions remains difficult and therefore further options are necessary. In addition to treating pain directly using pharmacologic and nonpharmacologic interventions, it is also important to treat comorbid conditions, such as major depressive disorder and anxiety, both of which are found to be strongly associated with chronic pain disorders. ${ }^{2}$ Chronic pain due to nonmalignant causes is believed to affect $9 \%$ of adults in the US, ${ }^{3}$ and approximately $20 \%$ of the world's population. ${ }^{4}$

Two types of nonmalignant chronic pain disorders are fibromyalgia and neuropathic pain. Neuropathic pain is reported by approximately $3 \%$ of the population at some time during their lives, ${ }^{5}$ and is characterized by tingling, burning, shock-like or shooting sensations, allodynia, and hyperalgesia. ${ }^{6,7}$ There are a number of disorders associated with neuropathic pain, the most common being diabetic neuropathy and lower back pain. Others include human immunodeficiency-associated pain, phantom pain, and multiple sclerosis. ${ }^{6-8}$ The pathophysiology of neuropathic pain is complex and numerous 
Table I Somatic and psychological manifestations of pain'

\begin{tabular}{ll}
\hline Physical attributes & Psychologic attributes \\
\hline Hypertension & Anxiety \\
Tachycardia & Depression \\
Diaphoresis & Fear \\
Mydriasis & Anger \\
Pallor & Insomnia \\
Gastrointestinal distress & \\
\hline
\end{tabular}

mechanisms may be involved. ${ }^{9}$ Perhaps due to the multiple etiologies of neuropathic pain, many types of drug classes are used in its treatment. Current recommendations for first-line therapy involve the use of tricyclic antidepressants, serotoninnorepinephrine reuptake inhibitors, antiepileptic drugs, and topical anesthetics. Second-line options involve the use of opioids and tramadol. ${ }^{8,10}$ Nonpharmacologic interventions are also used to treat neuropathies, and include techniques such as spinal cord stimulation, ${ }^{11}$ acupuncture, ${ }^{12}$ and psychologic interventions. $^{13}$

Fibromyalgia is an idiopathic, chronic, nonarticular pain syndrome, and is defined by The American College of Rheumatology as widespread pain in combination with tenderness at 11 of 18 specific tender point sites, with at least a threemonth duration. ${ }^{14}$ Patients will describe the pain as burning or gnawing, and also complain of stiffness. ${ }^{15}$ In addition to the musculoskeletal pain symptoms, patients often suffer from headaches, fatigue, mood and anxiety disorders, sleep disturbances, and cognitive disturbances. ${ }^{15,16}$ It is believed that approximately $0.5 \%-5.0 \%$ of the population suffers from fibromyalgia. ${ }^{17,18}$ Risk factors associated with a higher prevalence of fibromyalgia include female gender, increasing age, and belonging to a lower socioeconomic group. ${ }^{18}$

The pathophysiology of fibromyalgia is not fully understood, but compounds that increase serotonin and norepinephrine activity have proven efficacious in treating fibromyalgia. ${ }^{19}$ Duloxetine, a serotonin-norepinephrine reuptake inhibitor, is approved by the US Food and Drug Administration (FDA) for the treatment of painful diabetic neuropathy and fibromyalgia. This article will review the efficacy of duloxetine in the treatment of both of these chronic pain disorders.

\section{Duloxetine}

\section{Pharmacodynamics}

Duloxetine is a selective serotonin and norepinephrine reuptake inhibitor that is approved by the FDA for major depressive disorder, generalized anxiety disorder, diabetic peripheral neuropathic pain, and fibromyalgia. ${ }^{20}$
Its mechanism of action is through the blockade of reuptake of serotonin (5-HT) and norepinephrine. Studies show that duloxetine inhibits 5-HT and norepinephrine uptake in the hypothalamic synaptosomes of rats with a preference for 5 -HT. ${ }^{21}$

Platelet serotonin measurements can be used to show 5-HT reuptake inhibition due to the 5-HT reuptake function of platelets. An increase in whole blood 5-HT content represents 5-HT reuptake inhibition. Studies have shown a dose-dependent increase in whole blood 5-HT levels in patients taking duloxetine. ${ }^{22}$ Studies using positron emission tomography also indicate duloxetine's ability to inhibit 5-HT reuptake. One such study demonstrated serotonin transporter (5-HTT) occupancy in subjects dosed with duloxetine, thus establishing duloxetine's ability to block the reuptake of 5 - $\mathrm{HT}^{23}$

Several methods have been utilized to demonstrate norepinephrine reuptake inhibition by duloxetine. These include changes in urinary excretion of norepinephrine and metabolites, changes in cardiovascular effects, and monitoring of adverse event profiles. ${ }^{24} \mathrm{~A}$ decrease in whole body norepinephrine (norepinephrine plus its metabolites) in urinary excretion indicates decreased norepinephrine reuptake. Chalon et al demonstrated that there was a decrease in urinary excretion of norepinephrine metabolites, and not norepinephrine itself, in duloxetine treatment groups. ${ }^{25} \mathrm{An}$ increase in supine systolic blood pressure is also indicative of blocked norepinephrine reuptake. This was demonstrated in patients taking duloxetine $80 \mathrm{mg} /$ day. ${ }^{25}$

\section{Pharmacokinetics}

Oral duloxetine hydrochloride is well absorbed, with a time to peak plasma concentration of six hours. ${ }^{20}$ Food does not change the peak concentration, but will delay $\mathrm{T}_{\max }$ to up to 10 hours and slightly decreases total absorption. ${ }^{20}$ The steady state of duloxetine is reached in three days, independent of dose, with a mean terminal elimination half-life of 12.5 hours. ${ }^{26}$ Duloxetine pharmacokinetics are linear with respect to dose. ${ }^{27}$ The mean oral clearance based on the average of eight subjects was $114 \mathrm{~L} /$ hour, and apparent volume of distribution was 1943 L. ${ }^{27}$ Duloxetine is $90 \%$ protein-bound, ${ }^{20}$ therefore caution should be exercised when administering duloxetine concomitantly with medications that are highly protein-bound, such as warfarin and phenytoin.

Duloxetine metabolism and elimination is mainly through the liver, involving cytochrome (CYP) $1 \mathrm{~A} 2$ and CYP2D6 isoenzymes,${ }^{20}$ and has an extensive biotransformation through oxidation, methylation, and/or conjugation 
pathways. ${ }^{27}$ In a study using four subjects, urine collection was performed for 72 hours after duloxetine ingestion, and using high-pressure liquid chromatography/radiochemical detection, researchers found approximately $94 \%$ of the radioactivity excreted in the urine as the various duloxetine metabolites. The major plasma metabolite is the glucuronide conjugate of 4-hydroxy duloxetine. Unchanged duloxetine was undetectable in the urine. ${ }^{27}$

\section{Drug-drug interactions}

Duloxetine is metabolized by CYP1A2 and CYP2D6 to form the various metabolites excreted mainly in the urine. ${ }^{28}$ While duloxetine is a CYP2D6 substrate, it also is a moderately potent inhibitor of CYP2D6 and insignificant inhibitor of CYP1A2. ${ }^{20,28}$ Due to duloxetine's CYP2D6 inhibitory effect, care should be taken when coadministering other medications metabolized through this pathway. Furthermore, giving duloxetine with potent inhibitors of CYP2D6 and CYP1A2 should also be cautioned due to increases in duloxetine concentration. ${ }^{28}$ Several studies were done to examine the drug-drug interaction between duloxetine and CYP1A2 and CYP2D6 inhibitors and inducers. In two studies using extensive genotypic CYP2D6 metabolizers, subjects were given duloxetine with either desipramine (a CYP2D6 substrate), or paroxetine (a potent CYP2D6 inhibitor). ${ }^{29}$ In the duloxetine-desipramine study, concentrations of desipramine increased in the presence of duloxetine. In the duloxetineparoxetine study, duloxetine plasma concentrations increased moderately in the presence of paroxetine. Another potential drug interaction involves the use of anticoagulants (due to duloxetine's small 1A2 inhibition). ${ }^{25}$ Pharmacodynamic drug interactions may also occur with monoamine oxidase inhibitors and other serotonergic drugs, resulting in an increased risk of serotonin syndrome. An example of this involves an interaction between duloxetine and linezolid. Linezolid has been shown to be a nonselective inhibitor of monoamine oxidase.$^{30}$ It is recommended that patients be monitored closely if taking duloxetine concomitantly with any of these drug classes. ${ }^{20}$

\section{Efficacy in chronic pain}

A Medline search was done using the keywords "duloxetine", "Cymbalta ${ }^{\circledR} "$, "chronic pain", "neuropathic pain", "fibromyalgia", "efficacy", and "controlled trial". In addition, reference lists of relevant articles were thoroughly reviewed to determine additional sources of nonindexed published data. Randomized, double-blind, placebo-controlled trials of duloxetine for the treatment of either fibromyalgia or diabetic neuropathy were included for review. Eight Phase III controlled trials were found, five of which looked at the use of duloxetine in patients with fibromyalgia, and three looked at the use of duloxetine in patients with neuropathic pain. It is noteworthy that all data were based on industry-sponsored studies. The following section will summarize these trials, with each disorder being dealt with separately.

\section{Fibromyalgia}

Arnold et a ${ }^{31}$ conducted a randomized, multicenter, doubleblind, placebo-controlled trial to study the efficacy and tolerability of duloxetine in patients with fibromyalgia with or without current major depressive disorder. Study subjects were recruited from 18 sites in the US. The study consisted of a one-week, placebo lead-in phase followed by a 12-week, double-blind treatment phase. Subjects underwent a forced dose-titration phase for the first two weeks of treatment beginning with duloxetine $20 \mathrm{mg}$ daily, which was titrated upwards to a dose of $60 \mathrm{mg}$ twice daily. Primary outcome measures were defined by the Fibromyalgia Impact Questionnaire (FIQ) pain item and total score, with secondary outcomes including the FIQ items for fatigue, morning tiredness, and stiffness, number of tender points, and tender point pain threshold, the Clinical Global Impression of Severity (CGI-S) scale, the Patient Global Impression of Improvement (PGI-I) scale, and the Brief Pain Inventory (BPI) short form. Additionally, other secondary objectives sought to determine if the effects of duloxetine $60 \mathrm{mg}$ twice daily on the FIQ pain scores were dependent on whether or not the subject suffered from major depressive disorder, and also looked at overall quality of life. Regarding this, outcome measures were the Beck Depression Inventory-II scale, the Beck Anxiety Inventory (BAI) scale, the Medical Outcomes Study Short Form 36 (SF-36), the Quality of Life in Depression Scale, and the Sheehan Disability Scale.

A total of 207 subjects entered the treatment phase of the study, with 104 receiving active treatment and 103 receiving placebo. Both treatment arms reported a decrease in total FIQ score from baseline, with the duloxetine-treated group showing a significantly greater decrease in total FIQ score as compared with placebo at week 12 (mean \pm standard error [SE] for duloxetine was $-13.46 \pm 1.82$ versus $-7.93 \pm 1.73$ for placebo, $P=0.027)$. Differences in FIQ subscores for pain, fatigue, and morning tiredness between both treatment groups were not significant at 12 weeks. However, the difference in FIQ subscore for stiffness between both groups was significant at week 12 (mean $\pm \mathrm{SE}$ for duloxetine was $-2.33 \pm 0.30$ versus $-1.51 \pm 0.29$ for placebo, $P=0.048$ ). 
Both treatment groups experienced a reduction from baseline in number of tender points, as well as a reduction in mean tender point pain threshold, and in each case, those changes were significantly larger for the duloxetine group versus placebo. Differences between the two treatment groups in response rates, as defined by a $50 \%$ reduction in FIQ pain score, were not significant $(27.7 \%$ for duloxetine versus $16.7 \%$ for placebo, $P=0.06)$. Most of the SF-36 subscores, the Quality of Life in Depression Scale, and the Sheehan Disability Scale showed significantly greater improvements for the duloxetine group as compared with the placebo group. Secondary outcome measures that did not show significantly greater improvements for the duloxetine group versus placebo were the Beck Depression Inventory-II total score, the BAI total score, and the SF-36 mental, emotional role limit, and social functioning subscores.

A second study conducted by Arnold et al was a randomized, multicenter, double-blind, placebo-controlled trial which looked at the efficacy and tolerability of duloxetine in women with fibromyalgia with or without major depressive disorder. ${ }^{32}$ This study was conducted at 21 sites in the US, and consisted of a 12-week treatment phase whereby subjects were randomized to receive either duloxetine $60 \mathrm{mg}$ daily, duloxetine $60 \mathrm{mg}$ twice daily, or placebo. The primary outcome measure was the BPI (short form) 24-hour average pain severity score. Secondary outcomes were similar to those used in the previous study, except that this study included the BPI items for worst and least pain in the prior 24 hours, the FIQ total score, and the 17-item Hamilton Depression Rating Scale. A response was defined as a greater than $30 \%$ reduction in the BPI 24-hour average pain severity score.

A total of 354 women were enrolled into the treatment phase of the study and were randomly assigned to receive one of the three treatments, such that 118 subjects received duloxetine $60 \mathrm{mg}$ daily, 116 subjects received duloxetine $60 \mathrm{mg}$ twice daily, and 120 patients received placebo. A total of $138(39 \%)$ patients withdrew from the study during the treatment phase, but the rate of withdrawal was not significant between the three treatment groups $(P=0.407)$. However, the withdrawal rate was significant for active treatment groups versus placebo when measuring withdrawal rate due to adverse events.

For the primary outcome measure, there was a statistically significant improvement for both active treatments versus placebo. This improvement began at week 1 and continued through week 12, with the change from baseline of BPI average scores at week 12 being $-2.39 \pm 0.22$ for duloxetine $60 \mathrm{mg}$ daily $(P<0.001),-2.40 \pm 0.22$ for duloxetine
$60 \mathrm{mg}$ twice daily, and $-1.16 \pm 0.21$ for placebo. There was no significant difference between the two active treatment groups with regard to primary outcome.

The duloxetine $60 \mathrm{mg}$ daily group demonstrated significant improvements from baseline in all secondary outcome measures, with the exception of mean tender point pain threshold and number of tender points with low pain threshold. Likewise, for the duloxetine $60 \mathrm{mg}$ twice daily group, all secondary outcome measures showed significant improvements versus placebo, except for the Hamilton Depression Rating Scale. A response rate of $44 \%$ was observed for the duloxetine $60 \mathrm{mg}$ daily group $(P<0.001$ versus placebo), $43 \%$ for the duloxetine $60 \mathrm{mg}$ twice daily group ( $P<0.001$ versus placebo), and 19\% for placebo.

Two studies examined the efficacy and safety of duloxetine in fibromyalgia patients with or without major depressive disorder over a six-month period. Chappell ${ }^{33}$ conducted a double-blind, placebo-controlled study whereby patients were randomized to receive duloxetine $60-120 \mathrm{mg}$ daily or placebo. Outcome measures were similar to those in previous studies, with the coprimary outcome measures being change from baseline to endpoint in average pain item of the BPI at six months, plus improvements in the PGI-I from baseline to endpoint at six months. Patients' duloxetine doses were titrated upwards to $120 \mathrm{mg}$ if they had not had a $\geq 50 \%$ improvement in their BPI scores by week 13. A total of 330 subjects, of which over $90 \%$ were Caucasian females, were enrolled in the study, with 162 being randomized to active treatment group and 168 to placebo. Contrary to previous studies, there was no statistically significant difference in primary outcome between duloxetine-treated and placebo-treated subjects. For the coprimary outcome of change in average pain item of the BPI, there was a mean improvement of $-1.62 \pm 0.20$ for the duloxetine-treated group, and $-1.13 \pm 0.19$ for the placebo group $(P=0.053)$. For the coprimary outcome of mean change in PGI-I, there was an improvement of $3.43 \pm 0.13$ (SE) for the duloxetine group versus $3.72 \pm(0.12)$ for placebo group $(P=0.073)$. Many of the secondary outcome measures in this study also did not show significant differences between treatment groups, but there were significant differences between treatment groups in BPI least pain score and average interference, FIQ pain score, CGS-S, Multidimensional Fatigue Inventory (MFI) mental fatigue score, BDI-II total score, and AUC (area under the curve) of pain relief: (as derived from the BPI average pain score).

The other six-month study reported by Russell et al, ${ }^{34}$ had the same coprimary endpoints as the Chappell study. A total of 520 subjects were enrolled in the study, with 
278 completing the full six months of treatment. Subjects were randomly assigned in a 1:2:2:2 ratio to duloxetine $20 \mathrm{mg}$ once daily, $60 \mathrm{mg}$ once daily, $120 \mathrm{mg}$ once daily, or placebo. Subjects randomized to $60 \mathrm{mg}$ or $120 \mathrm{mg}$ had their doses titrated upwards from an initial dose of $30 \mathrm{mg}$ per day. The $20 \mathrm{mg}$ dose was used to determine the lowest effective dose of duloxetine. At three months, there were significant improvements in both coprimary outcome measures. The BPI average pain severity score saw an improvement of $-1.99 \pm 0.20$ for the duloxetine $60 \mathrm{mg} /$ day group, $-2.31 \pm 0.20$ for the duloxetine $120 \mathrm{mg} /$ day group, and $-1.39 \pm 0.20$ for placebo. Changes for both the $60 \mathrm{mg}$ and $120 \mathrm{mg}$ duloxetine groups were significant versus placebo, but the change observed in the $20 \mathrm{mg}$ /day duloxetine group $(-1.92 \pm 0.27)$ was not significant versus placebo. Improvements in PGI-I scores for all three active treatments showed statistical significance versus placebo (mean $\pm \mathrm{SE}$ for duloxetine $20 \mathrm{mg}$ was $2.85 \pm 18.82$, for duloxetine $60 \mathrm{mg}$ was $3.04 \pm 0.13$, for duloxetine $120 \mathrm{mg}$ was $2.89 \pm 0.13$, and for placebo was $3.39 \pm 0.13$ ). For the six-month endpoint, changes in BPI average pain severity score were significant for all active treatments versus placebo $(-2.22 \pm 0.28$ for the duloxetine $20 \mathrm{mg}$ group, $-19.98 \pm 0.21$ for duloxetine $60 \mathrm{mg}$, $-2.26 \pm 0.21$ for duloxetine $120 \mathrm{mg}$, and $-1.43 \pm 0.21$ for placebo). There were also significant improvements versus placebo for the duloxetine $20 \mathrm{mg}$ and $120 \mathrm{mg}$ groups in PGI-I score, but not for the $60 \mathrm{mg}$ group $(2.79 \pm 0.17$ for duloxetine $20 \mathrm{mg}, 3.08 \pm 0.13$ duloxetine $60 \mathrm{mg} ; P>0.05$ versus placebo, $2.93 \pm 0.13$ for duloxetine $120 \mathrm{mg}$, and $3.37 \pm 0.13$ for placebo). Similar to the six-month Chappell study, many of the secondary outcome measures did not reach statistical significance when comparing active treatments with placebo. The scores for CGI-S and MFI mental fatigue were the only secondary outcomes to reach significance versus placebo across all three active treatments. Interestingly, these outcomes were also statistically significant in the Chappell study. In addition to the scores for mental fatigue on the CGI-I and MFI, secondary outcomes that were significant versus placebo for the duloxetine $120 \mathrm{mg}$ group included all subsets of the MFI (except for the MFI general fatigue score), the SF-36 mental component summary, the BPI average pain severity score with and without major depressive disorder, and the PGI-I with major depressive disorder. For the duloxetine $60 \mathrm{mg}$ group, no further secondary outcomes reached significance, while for the duloxetine $20 \mathrm{mg}$ group, changes in the EuroQol EQ-5D and PGI-I without major depressive disorder were statistically significant versus placebo.
A fifth study that examined the long-term (52-week) efficacy and safety of duloxetine in patients with fibromyalgia was reported by Chappell et al. ${ }^{35}$ This was a randomized, multicenter Phase III study conducted at 33 centers internationally, and consisted of an eight-week, open-label phase followed by a 52 -week, double-blind phase. Its primary goal was to evaluate the safety and tolerability of long-term duloxetine use in patients with fibromyalgia, but efficacy measures were used as secondary outcomes. Efficacy measures included the BPI-Modified Short Form, FIQ total score, PGI-I and CGI-S scores, number of tender points with a low threshold, mean of the 18 tender points pain threshold, and the Sheehan Disability Scale global functional impairment score. A response was defined as a greater than $50 \%$ reduction in BPI average pain score from baseline to endpoint during the open-label period.

A total of 350 fibromyalgia patients were enrolled in the open-label phase and were treated with duloxetine $60 \mathrm{mg}$ daily for eight weeks. Of the 350 subjects, 307 continued to the double-blind phase where they were randomized to receive either duloxetine $60 \mathrm{mg}$ daily or duloxetine $120 \mathrm{mg}$ daily for 52 weeks. In the initial open-label phase, 34.8\% of patients responded to therapy, and all efficacy outcome measures showed a statistically significant improvement from baseline. At the end of the double-blind period, there were significant differences between both treatment groups in a number of the efficacy outcome measures. These included changes from baseline (defined as assessments taken at the end of the open-label phase) in the BPI interference scores for walking ability, sleep, enjoyment of life, and average interference, with the $60 \mathrm{mg}$ group showing greater improvements than the $120 \mathrm{mg}$ group. The duloxetine $60 \mathrm{mg}$ group also showed significant improvements from baseline versus the $120 \mathrm{mg}$ group for the mean PGI-I score, FIQ total score, mean tender point threshold, number of tender points with low threshold, and the Sheehan Disability Scale global functional impairment total score. Persistence of effect of duloxetine in pain reduction was assessed in those patients who had achieved a response and had continued to be treated with duloxetine $60 \mathrm{mg}$ daily (36 subjects). In this case, persistence of effect was not demonstrated. Table 2 contains a summary of the above studies.

\section{Neuropathic pain}

Goldstein et $\mathrm{al}^{36}$ reported on a 12 week, multicenter, parallelgroup, double-blind, randomized, placebo-controlled study of duloxetine use in patients diagnosed with diabetic neuropathy. Pain had to have been present for at least six months 
Table 2 Efficacy of duloxetine in treating pain in fibromyalgia patients

\begin{tabular}{|c|c|c|c|c|c|c|c|c|}
\hline \multirow[t]{3}{*}{ Study duration } & \multicolumn{8}{|l|}{ Study } \\
\hline & \multicolumn{2}{|l|}{ Arnold et $\mathbf{a l}^{31}$} & \multicolumn{3}{|l|}{ Arnold et $\mathrm{al}^{32}$} & \multicolumn{3}{|l|}{ Russell et $\mathbf{a l}^{34}$} \\
\hline \multirow{2}{*}{\multicolumn{9}{|c|}{ Baseline demographics }} \\
\hline & & & & & & & & \\
\hline Percentage female & 89 & & 100 & & & 94.8 & & \\
\hline Mean age (years) & 49 & & 49.6 & & & 51 & & \\
\hline Treatment group & DLX $120 \mathrm{mg}$ & PBO & DLX $60 \mathrm{mg}$ & $\mathrm{DLX} 120 \mathrm{mg}$ & PBO & $\mathrm{DLX} 20 \mathrm{mg}$ & $\mathrm{DLX} 60 \mathrm{mg}$ & DLX $120 \mathrm{mg}$ \\
\hline $\mathrm{n}$ & 104 & 103 & 118 & 116 & 120 & 79 & 150 & 147 \\
\hline \multicolumn{9}{|c|}{ Outcome measure (change from baseline $\pm \mathrm{SE}$ ) } \\
\hline FIQ total score & $-13.46(1.82)$ & $-7.93(1.73)$ & $-16.72(1.53)$ & $-16.81(1.54)$ & $-8.35(1.53)$ & $-14.60(1.83)^{*}$ & $-15.41(1.40)^{*}$ & $-14.50(1.38)^{*}$ \\
\hline $\begin{array}{l}\text { Mean tender point } \\
\text { pain threshold }\end{array}$ & $0.29(0.07)$ & $-0.04(0.07)$ & $0.22(0.08)$ & $0.39(0.08)$ & $0.06(0.08)$ & $0.5 I(0.1 I)$ & $0.52(0.08)$ & $0.42(0.08)$ \\
\hline \multicolumn{9}{|l|}{ Brief pain inventory } \\
\hline Average pain & $-1.83(0.24)$ & $-0.94(0.23)$ & $-2.39(0.22)$ & $-2.40(0.22)$ & $-1.16(0.21)$ & $-1.92(0.27)$ & $-1.99(0.2)^{*}$ & $-2.31(0.20)^{*}$ \\
\hline Worst pain & - & - & $-2.53(0.25)$ & $-2.37(0.25)$ & $-1.35(0.24)$ & - & - & - \\
\hline Least pain & - & - & $-1.77(0.20)$ & $-1.76(0.20)$ & $-0.58(0.20)$ & - & - & - \\
\hline CGI-S & $-0.72(0.12)$ & $-0.39(0.12)$ & $-0.84(0.10)$ & $-0.84(0.10)$ & $-0.44(0.10)$ & $-0.96(0.12)$ & $-1.06(0.10)^{*}$ & $-1.10(0.09)^{*}$ \\
\hline PGI-I & $3.02(0.17)$ & $3.53(0.17)$ & $3.11(1.77)$ & $3.06(1.73)$ & $3.71(1.5)$ & $2.85(0.17)^{*}$ & $3.04(-0.13)^{*}$ & $2.89(0.13)^{*}$ \\
\hline
\end{tabular}

Notes: *Results were statistically significant $(P<0.05)$ versus placebo.

Abbreviations: SE, standard error; DLX, duloxetine; PBO, placebo; FIQ, Fibromyalgia Impact Questionnaire; CGI-I, Clinical Global Impressions of Severity; PGI-I, Patient's Global Impressions of Improvement.

prior to the onset of the study, and rate a score of at least 3 on the Michigan Neuropathy Screening Instrument. Subjects were randomized in a 1:1:1:1 fashion to receive duloxetine $20 \mathrm{mg}$ daily, duloxetine $60 \mathrm{mg}$ daily, duloxetine $120 \mathrm{mg}$ daily (given as $60 \mathrm{mg}$ twice daily), or placebo for 12 weeks. The primary outcome measure was the mean change from baseline to endpoint on the 24-hour Average Pain Severity score. Secondary outcome measures included the Average Daily Severity and Average Night Severity pain scores, Worst Pain Severity score, BPI interference and severity scores, CGI-S, PGI-I, SF-36, EuroQol EQ-5D, the Beck Depression Inventory-II (BDI-II), and the BAI.

A total of 457 subjects were enrolled in the study, the majority of whom had Type 2 diabetes mellitus (88.4\%), and had been diagnosed with diabetic neuropathy for a mean duration of 3.7 years, of whom 344 subjects completed the study. The primary outcome measure of mean change from baseline in 24-hour Average Pain Severity Score was statistically significant for the duloxetine $60 \mathrm{mg}$ and duloxetine $120 \mathrm{mg}$ groups versus placebo (mean change \pm SE for duloxetine $60 \mathrm{mg}$ was $-2.89 \pm 0.22$, for duloxetine $120 \mathrm{mg}$ was $-3.24 \pm 0.23$, and for placebo was $-1.91 \pm 0.22$ ). A $50 \%$ reduction in 24-hour Average Pain Severity Score from baseline to endpoint was achieved by $41 \%$ in the duloxetine $20 \mathrm{mg}$ group, $49 \%$ in the $60 \mathrm{mg}$ group, $52 \%$ in the $120 \mathrm{mg}$ group, and $26 \%$ in the placebo group $(P<0.05$ for all active treatments versus placebo). All secondary outcome measures met statistical significance for the duloxetine $120 \mathrm{mg}$ group, with the exception of the Dynamic Allodynia Severity score and the BAI score. For the duloxetine $60 \mathrm{mg}$ group, there were also significant improvements versus placebo in secondary outcome measures of pain (an exception to this was the Dynamic Allodynia Severity score), but many of the depression and anxiety outcome measures did not show significant improvement versus placebo. For the duloxetine $20 \mathrm{mg}$ group, two secondary outcomes (CGI-S score, and SF McGill total score) showed greater improvement versus placebo. Further analysis of the data gathered in this study demonstrated that pain reduction in the duloxetine $60 \mathrm{mg}$ group was $94.8 \%$, attributable to a direct effect of the drug on pain symptoms (as opposed to a $0.2 \%$ indirect effect through mood improvement, and a 5.1\% indirect effect through improvement in anxiety). This was also reflected in the duloxetine $120 \mathrm{mg}$ group, which showed that improvements in pain symptoms were $88.6 \%$ due to a direct effect, and $11.4 \%$ due to indirect effects.

Another 12-week trial studying the use of duloxetine in treating neuropathic pain was reported on by Raskin et al. ${ }^{37}$ The study design was similar to the Goldstein et al study, except that this study did not include a duloxetine $20 \mathrm{mg}$ group. There were 348 patients enrolled in the study, with 116 being randomized into each of the following treatment groups: duloxetine $60 \mathrm{mg}$ daily, duloxetine $120 \mathrm{mg}$ daily (60 mg twice daily), and placebo. The primary outcome measure was the change in weekly mean of the 24-hour pain scores. Secondary outcome measures were also very similar 


\begin{tabular}{|c|c|c|c|c|c|c|c|c|}
\hline & & & & & \multicolumn{2}{|l|}{ Chappell $^{33}$} & \multicolumn{2}{|c|}{ Chappell et al $\left.\right|^{35}$} \\
\hline & 6 months & & & & 6 months & & 12 months & \\
\hline & & & & & 93.3 & & 95.7 & \\
\hline & & & & & 50.5 & & 49 & \\
\hline РBO & $\mathrm{DLX} 20 \mathrm{mg}$ & $\mathrm{DLX} 60 \mathrm{mg}$ & DLX 120 mg & PBO & $\mathrm{DLX} 60 / / 20 \mathrm{mg}$ & PBO & $\mathrm{DLX} 60 \mathrm{mg}$ & DLX $120 \mathrm{mg}$ \\
\hline 144 & 49 & 97 & 95 & 84 & 162 & 168 & 104 & 203 \\
\hline$-10.05(1.42)$ & $-14.77(1.88)$ & $-12.28(1.44)$ & $-13.86(1.41)$ & $-10.42(1.46)$ & $-7.96(1.35)$ & $-5.81(1.29)$ & $-0.69(1.82)$ & $3.49(1.34)^{*}$ \\
\hline $0.33(0.08)$ & $0.54(0.12)$ & $0.52(0.09)$ & $0.54(0.09)$ & $0.42(0.09)$ & $0.40(0.09)$ & $0.18(0.08)$ & $0.58(0.11)$ & $0.32(0.08)^{*}$ \\
\hline$-1.39(0.20)$ & $-2.22(0.28) *$ & $-1.98(0.21)^{*}$ & $-2.26(0.21)^{*}$ & $-1.43(0.2 I)$ & $-1.62(0.20)$ & $-1.13(0.19)$ & $-0.37(0.26)$ & $-0.16(0.19)$ \\
\hline- & - & - & - & - & $-1.75(0.22)$ & $-1.25(0.21)$ & $-0.53(0.28)$ & $-0.18(0.21)$ \\
\hline- & - & - & - & - & $-1.22(0.19)$ & $-0.73(0.18)$ & $-0.12(0.26)$ & $0.21(0.19)$ \\
\hline $0.70(0.10)$ & $-0.97(0.13)^{*}$ & $-1.07(0.10)^{*}$ & $-1.14(0.10)^{*}$ & $-0.66(0.10$ & $-0.57(0.09)$ & $-0.28(0.08)$ & $-0.23(0.11)$ & $-0.04(0.08)$ \\
\hline $3.39(0.13)$ & $2.79(0.17)^{*}$ & $3.08(0.13)$ & $2.93(0.13)^{*}$ & $3.37(0.13)$ & $3.43(0.13)$ & $3.72(0.12)$ & $2.19(0.15)$ & $2.65(0.11)^{*}$ \\
\hline
\end{tabular}

to those used in the Goldstein et al study. A response was defined as a $30 \%$ reduction from baseline in the primary outcome measure.

All outcome measures, both primary and secondary, showed statistically significant improvements versus placebo for the duloxetine $120 \mathrm{mg}$ group, with exception of mean change from baseline in HAM-D score and Dynamic Allodynia Severity score. The duloxetine $60 \mathrm{mg}$ group also showed significant improvements over placebo with regard to most of the outcome measures with the exceptions of the HAM-D score, Dynamic Allodynia Severity score, and the BPI scores for mood and relationships. For the primary outcome measure, mean change from baseline for the duloxetine $60 \mathrm{mg}$ group was $-2.50 \pm 0.18$, for the duloxetine $120 \mathrm{mg}$ group was $-2.47 \pm 0.18$, and for the placebo was $-1.60 \pm 0.18$. Response rates were superior to placebo for active treatments, ie, $68.14 \%$ for duloxetine $60 \mathrm{mg}, 64.04 \%$ for duloxetine $60 \mathrm{mg}$, and $43.36 \%$ for placebo $(P<0.05)$. A path analysis resulted in a similar outcome to that of the Goldstein study in that the effect of duloxetine on pain reduction was due to a $98.0 \%$ direct effect for the duloxetine $120 \mathrm{mg}$ group and $92.7 \%$ for the duloxetine $60 \mathrm{mg}$ group.

The third study which examined the efficacy of duloxetine in treating diabetic neuropathic pain was reported by Wernicke et al. ${ }^{38}$ This was a 12 -week study with a similar study design to the Raskin et al study, ie, subjects were randomly assigned to either placebo, duloxetine $60 \mathrm{mg}$ daily, or duloxetine $120 \mathrm{mg}$ daily (given as twice-daily doses of $60 \mathrm{mg}$ ). As in the previous studies, the primary outcome measure was the reduction in the weekly mean 24-hour pain scores. A response was again defined as a decrease of $30 \%$ from baseline to endpoint in the 24-hour average pain score. Secondary outcome measures were similar to those in the previous two studies. A total of 334 subjects were randomized to receive treatment, with 108 receiving placebo, 114 receiving duloxetine $60 \mathrm{mg}$ daily, and 112 receiving duloxetine $60 \mathrm{mg}$ twice daily. Enrolled patients had suffered from diabetes for a mean of 10.2 years, and their average age was 60.7 years.

The primary outcome measure for both active treatments demonstrated significant improvement versus placebo, with the mean change from baseline to week 12 for the 24-hour average pain score being $-2.84 \pm 0.23$ for duloxetine $120 \mathrm{mg}$ daily, $-2.72 \pm 0.22$ for duloxetine $60 \mathrm{mg}$ daily, and $-1.39 \pm 0.23$ for placebo. Secondary outcome measures that demonstrated significant improvements versus placebo for both active treatment groups included BPI pain severity scores for average pain, worst pain, least pain, and pain right now, CGI-S scores, PGI-I scores, and the Short Form of the McGill Pain Questionnaire scores. In addition, there was a significant improvement in HAMD-17 score for the duloxetine $120 \mathrm{mg}$ daily group, but not for the duloxetine $60 \mathrm{mg}$ daily group. The secondary endpoint of change in Dynamic Allodynia Severity score from baseline to endpoint showed no statistical difference versus placebo for either of the active treatments. The above studies are summarized in Table 3. 


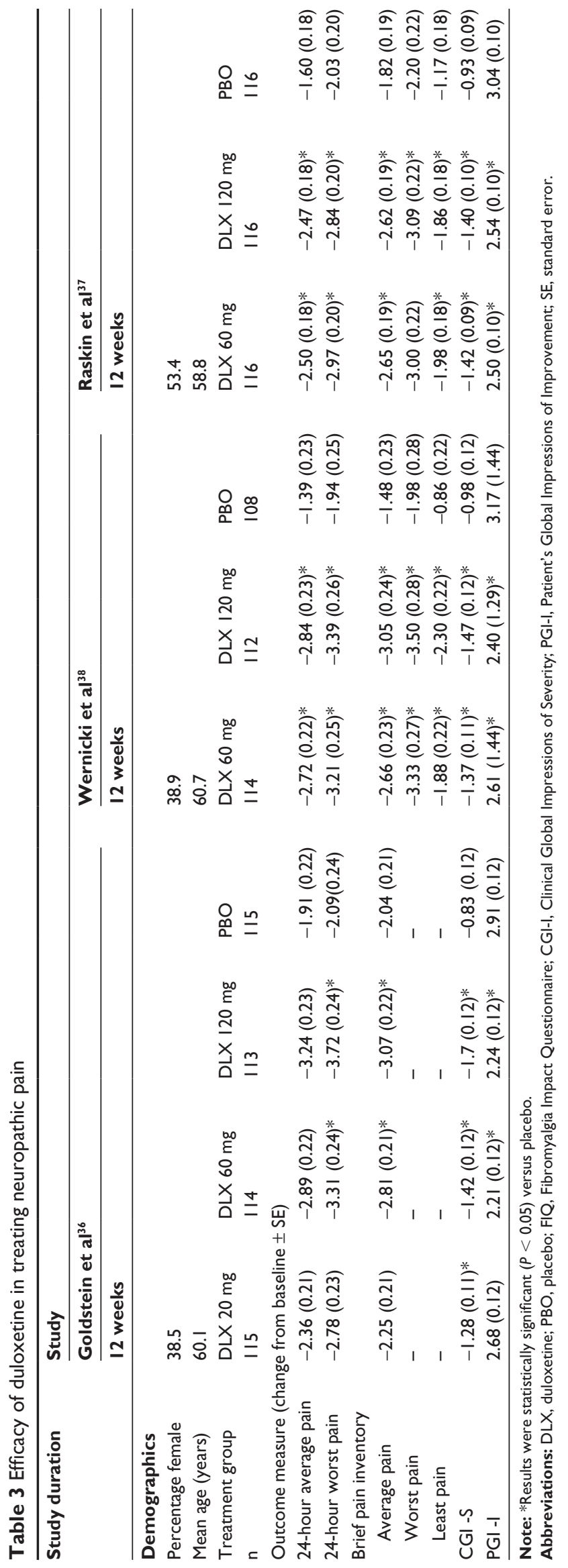

\section{Safety and tolerability}

In general, duloxetine was safe and well tolerated, with adverse events being mild to moderate in severity. The most common adverse event was nausea (experienced by about $30 \%-45 \%$ of duloxetine patients) and was the most frequent reason for study discontinuation due to an adverse event. Other adverse events that occurred more often than placebo were constipation, dry mouth, somnolence, sweating, changes in appetite, insomnia, and dizziness, although these were regarded as mild and occurred at a relatively low rate. ${ }^{31-34,38}$

Serious adverse events occurred at a relatively low frequency, and consisted of appendicitis, and a blood creatine phosphokinase and hepatic enzyme increase, ${ }^{32}$ arthralgia, gait disturbance, pseudomonal lung infection, muscular weakness, paresthesias, and pseudoneurologic symptoms, ${ }^{33}$ asthma and suicidal ideation, ${ }^{34,35}$ atrial fibrillation, cholecystitis, diabetes, nephrolithiasis, urinary calculi, and ventricular extrasystoles, ${ }^{37}$ congestive cardiac failure, coronary artery stenosis, and electrolyte embalances,$^{38}$ chest pain, hyperglycemia, and myocardial infarction. ${ }^{36}$ There was no significant difference in serious adverse events between the active and placebo groups. There was one fatality, although this was determined to be unrelated to the study drug. ${ }^{36}$ Discontinuation-emergent adverse events occurred at a rate of $1 \%$ to $3 \%$, and included dizziness, vomiting, crying, nausea, and vertigo, with vomiting being the most prominent $(3.5 \%){ }^{35}$

There were differences in rates of discontinuation between groups with regard to dose of duloxetine, with the higher doses typically having higher rates of discontinuation. For example, in the Goldstein et al study, ${ }^{36}$ discontinuation rates were $4.3 \%$ for the duloxetine $20 \mathrm{mg}$ group, $13.2 \%$ for the $60 \mathrm{mg}$ group, and $19.5 \%$ for the $120 \mathrm{mg}$ group. In the Chappel1 ${ }^{33}$ study, 7.45 of patients randomized to duloxetine withdrew due to lack of efficacy versus $14.9 \%$ of placebo patients $(P=0.036)$.

\section{Discussion}

Duloxetine is approved for the treatment for fibromyalgia and diabetic peripheral neuropathic pain at doses of $60 \mathrm{mg}$ daily. ${ }^{20}$ Several controlled studies have indicated that duloxetine can reduce pain symptoms in patients diagnosed with fibromyalgia or neuropathic pain. Duloxetine demonstrated significant reductions in FIQ and BPI scores and other measures of pain in fibromyalgia patients, and reductions in 24-hour average pain severity score in diabetic neuropathy patients. Duloxetine also appears to be relatively well tolerated, with adverse events being relatively mild and comparable with 
other reuptake inhibitors. Interestingly, when a path analysis (a statistical analysis that determines the degree of dependency between variables) was conducted, it was found that improvements in pain symptoms were due to a direct effect of the duloxetine, rather than an indirect effect caused by improvements in comorbid conditions, such as depression, for example. This analysis is further reinforced by the relatively small improvements seen in depressive symptoms in many of the studies when compared with placebo.

In general, tolerability seems to be dose-dependent, therefore, the recommended dose of duloxetine in the treatment for chronic pain due to fibromyalgia and peripheral neuropathy is $60 \mathrm{mg}$ once daily. This daily dose also represents the lowest consistently effective dose. Some patients may have additional benefit from doses up to $60 \mathrm{mg}$ twice daily, although tolerability may be an issue. The adverse effects most frequently seen with the use of duloxetine treatment include nausea, dizziness, headache, and constipation, and may occur more frequently with higher doses. A dose titration is recommended to increase tolerability and decrease initial adverse events. More information on the safety and tolerability of duloxetine in the treatment of pain can be found elsewhere. ${ }^{39}$ Drug interactions with duloxetine are minimal due to only a weak inhibition of CYP450 2D6, although predictable effects mediated by 5-HT remain, and therefore caution should be used when other serotonergic medications are being used concomitantly, especially monoamine oxidase inhibitors. Prescribing precautions for patients taking duloxetine include monitoring of hepatotoxicity due to increased risk of serum transaminases. For this reason, caution is advised in patients with a strong history of alcohol use due to potential for combined liver injury. ${ }^{40}$

There have been no controlled noninferiority comparator studies done with duloxetine, therefore it is difficult to assess whether duloxetine is a more appropriate treatment option compared with more established and less costly alternatives. Other treatment options available for treating chronic pain due to fibromyalgia or diabetic neuropathy involve the use of many different classes of pharmacologic agents, including tricyclic antidepressants, selective serotonin reuptake inhibitors and norepinephrine reuptake inhibitors, anticonvulsants, and opiates. ${ }^{39}$ Anticonvulsants, such as gabapentin and pregabalin, are effective at reducing pain, making them reasonable first-line options for the patient suffering from chronic neuropathic pain. Opioid use in treating fibromyalgia is controversial, with the current evidence for their use being inconclusive. ${ }^{10,41}$ In addition, opiates have a high potential for adverse effects and there is always the risk of tolerance and abuse. Antidepressants, such as the selective serotonin reuptake inhibitor, fluoxetine, have been shown to be modestly effective in treating chronic pain associated with fibromyalgia. However, tricyclic antidepressants and norepinephrine reuptake inhibitors, such as duloxetine and milnacipran (a norepinephrine reuptake inhibitor indicated only for the treatment of fibromyalgia by the FDA) seem to have better clinical utility. Their efficacy in pain reduction is also believed to be associated with a direct effect of the drug rather than the drug's ability to reduce depressive or anxiety symptoms. ${ }^{41}$ Duloxetine is one additional option available from the medications in the antidepressant class. It should also be noted, that there are no direct comparator studies between these different drug classes, thus it is difficult to ascertain the superiority of one treatment over another.

\section{Disclosure}

All efficacy studies reported in this article were sponsored by Eli Lilly, who developed and market duloxetine (Cymbalta $\left.{ }^{\circledR}\right)$. Otherwise, the authors report no conflicts of interest in this work.

\section{References}

1. American Pain Society. Principles of Analgesic Use in the Treatment of Acute Pain and Cancer Pain. 6th ed. Glenview, IL: American Pain Society; 2008.

2. von Korff M, Simon G. The relationship between pain and depression. Br J Psychiatry Suppl. 1996;(30):101-108.

3. Roper Starch Worldwide Inc for the American Academy of Pain Medicine, American Pain Society, and Janssen Pharmaceuticals. Chronic Pain in America: Roadblocks to Relief, 1999. Available from: http:// www.ampainsoc.org/links/roadblocks/. Accessed on 2010 Nov 5.

4. Gureje O, Von Korff M, Simon GE, Gater R. Persistent pain and well-being: A World Health Organization study in primary care. JAMA. 1998; 280(2):147-151.

5. Turk DC, Audette J, Levy RM, Mackey SC, Stanos S. Assessment and treatment of psychosocial comorbidities in patients with neuropathic pain. Mayo Clin Proc. 2010;85 Suppl 3:S42-S50.

6. Woolf CJ, Mannion RJ. Neuropathic pain: Aetiology, symptoms, mechanisms, and management. Lancet. 1999;353(9168):1959-1964.

7. Baron R. Mechanisms of disease: Neuropathic pain - a clinical perspective. Nat Clin Pract Neurol. 2006;2(2):95-106.

8. O'Connor AB, Dworkin RH. Treatment of neuropathic pain: An overview of recent guidelines. Am J Med. 2009;122 Suppl 10:S22-S32.

9. Oaklander AL. Mechanisms of pain and itch caused by herpes zoster (shingles). J Pain. 2008;9(1 Suppl 1):S10-S18.

10. Veves A, Backonja M, Malik RA. Painful diabetic neuropathy: Epidemiology, natural history, early diagnosis, and treatment options. Pain Med. 2008;9(6):66-74

11. de Leon-Casasola OA. Spinal cord and peripheral nerve stimulation techniques for neuropathic pain. J Pain Symptom Manage. 2009; 38 Suppl 2:S28-S38.

12. Abuaisha BB, Costanzi JB, Boulton AJ. Acupuncture for the treatment of chronic painful peripheral diabetic neuropathy: A long-term study. Diabetes Res Clin Pract. 1998;39(2):115-121.

13. Adams N, Poole H, Richardson C. Psychological approaches to chronic pain management: Part 1. J Clin Nurs. 2006;15(3):290-300. 
14. Wolfe F, Smythe HA, Yunus MB, et al. The American College of Rheumatology 1990 Criteria for the Classification of Fibromyalgia. Report of the Multicenter Criteria Committee. Arthritis Rheum. 1990;33(2): 160-172.

15. Chakrabarty S, Zoorob R. Fibromyalgia. Am Fam Physician. 2007; 76(2):247-254.

16. Arnold LM, Hudson JI, Keck PE, et al. Comorbidity of fibromyalgia and psychiatric disorders. J Clin Psychiatry. 2006;67(8):1219-1225.

17. Wolfe F, Ross K, Anderson J, Russell IJ, Hebert L. The prevalence and characteristics of fibromyalgia in the general population. Arthritis Rheum. 1995;38(1):19-28.

18. White KP, Harth M. Classification, epidemiology, and natural history of fibromyalgia. Curr Pain Headache Rep. 2001;5(4):320-329.

19. Arnold LM, Keck PE, Welge JA. Antidepressant treatment of fibromyalgia. A meta-analysis and review. Psychosomatics. 2000;41(2): 104-113.

20. Cymbalta ${ }^{\circledR}$ [Package insert]. Indianapolis, IN: Eli Lilly and Company; 2009.

21. Kasamo K, Blier P, de Montigny C. Blockade of the serotonin and norepinephrine uptake processes by duloxetine: In vitro and in vivo studies in the rat brain. J Pharmacol Exp Ther. 1996;277(1):278-286.

22. Turcotte JE, Debonnel G, de Montigny C, Hébert C, Blier P. Assessment of the serotonin and norepinephrine reuptake blocking properties of duloxetine in healthy subjects. Neuropsychopharmacology. 2001;24(5):511-521.

23. Takano A, Suzuki K, Kosaka J, et al. A dose-finding study of duloxetine based on serotonin transporter occupancy. Psychopharmacology (Berl). 2006;185(3):395-399.

24. Trivedi MH, Desaiah D, Ossanna MJ, et al. Clinical evidence for serotonin and norepinephrine reuptake inhibition of duloxetine. Int Clin Psychopharmacol. 2008;23(3):161-169.

25. Chalon SA, Granier L, Vandenhende FR, et al. Duloxetine increases serotonin and norepinephrine availability in healthy subjects: A doubleblind, controlled study. Neuropsychopharmacology. 2003;28(9): $1685-1693$.

26. Sharma A, Goldberg MJ, Cerimele BJ. Pharmacokinetics and safety of duloxetine, a dual-serotonin and norepinephrine reuptake inhibitor. J Clin Pharmacol. 2000;40(2):161-167.

27. Lantz RJ, Gillespie TA, Rash TJ, et al. Metabolism, excretion, and pharmacokinetics of duloxetine in healthy human subjects. Drug Metab Dispos. 2003;31(9):1142-1150.

28. Spina E, Santoro V, D'Arrigo C. Clinically relevant pharmacokinetic drug interactions with second-generation antidepressants: An update. Clin Ther. 2008;30(7):1206-1227.
29. Skinner MH, Kuan H, Pan A, et al. Duloxetine is both an inhibitor and a substrate of cytochrome P4502D6 in healthy volunteers. Clin Pharmacol Ther. 2003;73(3):170-177.

30. Strouse TB, Kerrihard TN, Forscher CA, Zakowski P. Serotonin syndrome precipitated by linezolid in a medically ill patient on duloxetine. J Clin Psychopharmacol. 2006;26(6):681-683.

31. Arnold LM, Lu Y, Crofford LJ, et al. A double-blind, multicenter trial comparing duloxetine with placebo in the treatment of fibromyalgia patients with or without major depressive disorder. Arthritis Rheum. 2004;50(9):2974-2984.

32. Arnold LM, Rosen A, Pritchett YL, et al. A randomized, double-blind, placebo-controlled trial of duloxetine in the treatment of women with fibromyalgia with or without major depressive disorder. Pain. 2005;119(1-3):5-15.

33. Chappell AS. A six-month double blind, placebo-controlled, randomized clinical trial of duloxetine for the treatment of fibromyalgia. Int $J$ Gen Med. 2008;1:91-102.

34. Russell IJ, Mease PJ, Smith TR, et al. Efficacy and safety of duloxetine for treatment of fibromyalgia in patients with or without major depressive disorder: Results from a 6-month, randomized, double-blind, placebo-controlled, fixed-dose trial. Pain. 2008;136(3): 432-444.

35. Chappell AS, Littlejohn G, Kajdasz DK, et al. A 1-year safety and efficacy study of duloxetine in patients with fibromyalgia. Clin J Pain. 2009;25(5):365-375.

36. Goldstein DJ, Lu Y, Detke MJ, Lee TC, Iyengar S. Duloxetine vs placebo in patients with painful diabetic neuropathy. Pain. 2005;116(1-2): 109-118.

37. Raskin J, Pritchett YL, Wang F, et al. A double-blind, randomized multicenter trial comparing duloxetine with placebo in the management of diabetic peripheral neuropathic pain. Pain Med. 2005;6(5):346-356.

38. Wernicke JF, Pritchett YL, D'Souza DN, et al. A randomized controlled trial of duloxetine in diabetic peripheral neuropathic pain. Neurology. 2006;67(8):1411-1420.

39. Lunn MP, Hughes RA, Wiffen PJ. Duloxetine for treating painful neuropathy or chronic pain. Cochrane Database Syst Rev. 2009;(4): CD007115

40. Barkin RL, Barkin S. The role of venlafaxine and duloxetine in the treatment of depression with decremental changes in somatic symptoms of pain, chronic pain, and the pharmacokinetics and clinical considerations of duloxetine pharmacotherapy. Am J Ther. 2005;12(5):431-438.

41. Sumpton JE, Moulin DE. Fibromyalgia: Presentation and management with a focus on pharmacological treatment. Pain Res Manag. 2008;13(6):477-483.
Journal of Pain Research

\section{Publish your work in this journal}

The Journal of Pain Research is an international, peer-reviewed, open access, online journal that welcomes laboratory and clinical findings in the fields of pain research and the prevention and management of pain. Original research, reviews, symposium reports, hypothesis formation and commentaries are all considered for publication.

\section{Dovepress}

The manuscript management system is completely online and includes a very quick and fair peer-review system, which is all easy to use. Visit http://www.dovepress.com/testimonials.php to read real quotes from published authors. 